\title{
TAIGA - an advanced hybrid detector complex for astroparticle physics, cosmic ray physics and gamma-ray astronomy.
}

\author{
N. Budnev ${ }^{\mathrm{a}, *}$, L. Kuzmichev ${ }^{b}$, R. Mirzoyan ${ }^{c}$, on behalf of the TAIGA Collaboration \\ (a complete list of authors can be found at the end of the proceedings) \\ a Institute of Applied Physics ISU, Irkutsk, Russia \\ b Skobeltsyn Institute of Nuclear Physics MSU, Moscow, Russia \\ c Max-Planck-Institute for Physics, Munich, Germany \\ E-mail:nbudneveapi.isu.ru,kuzedecl.sinp.msu.ru, \\ razmik.mirzoyanempp.mpg.de
}

The physical motivations and performance of the TAIGA (Tunka Advanced Instrument for cosmic ray physics and Gamma Astronomy) project are presented. The TAIGA observatory addresses ground-based gamma-ray astronomy and astroparticle physics at energies from a few $\mathrm{TeV}$ to several $\mathrm{PeV}$, as well as cosmic ray physics from $100 \mathrm{TeV}$ to several EeV. The pilot TAIGA complex is located in the Tunka valley, $\sim 50 \mathrm{~km}$ West from the southern tip of the lake Baikal. It includes the air Cherenkov light integrating TAIGA-HiSCORE array with 120 wideangle optical stations distributed over on area of $\sim 1 \mathrm{~km}^{2}$ and three $4-\mathrm{m}$ class Imaging Atmospheric Cherenkov Telescopes of the TAIGA-IACT array. The latter array has a shape of a triangle with side lengths of about $300 \mathrm{~m}, 400 \mathrm{~m}$ and $500 \mathrm{~m}$. The expected integral sensitivity of the $1 \mathrm{~km}^{2}$ TAIGA detector will be about $2,5 \times 10^{-13} \mathrm{TeV} \mathrm{cm}^{-2} \mathrm{sec}^{-1}$ for detection of $\mathrm{E} \geq 100 \mathrm{TeV}$ gamma-rays in 300 hours of observations. The combination of the wide angle Cherenkov array with IACTs offers a cost-effective way to build a really large (up to $10 \mathrm{~km}^{2}$ ) array for very high energy gamma-ray astronomy. The reconstruction of the given EAS energy, incoming direction and the core position, from the TAIGA-HiSCORE data, allows one to increase the distance between the relatively expensive IACTs of to $600-800 \mathrm{~m}$. These, together with the surface and underground electron/muon detectors will be used for selection of gamma-ray induced EAS. Present status of the project, together with the current array description and the first experimental results and plans for the future are presented. 


\section{Introduction}

In recent years, gamma-ray astronomy became one of the most dynamically developing fields of astroparticle physics. Imaging Atmospheric Cherenkov Telescope (IACT) arrays, H.E.S.S. [1], MAGIC [2] and VERITAS [3] discovered more than 200 sources with gamma-ray energy spectra typically extending from a few hundreds of Gev, up to several tens of TeV. The Tibet collaboration reported about detection of 24 photon-like events with $\mathrm{E}>100 \mathrm{TeV}$ against 5.5 background events from Crab Nebula [4]. The LHAASO collaboration using the first several months of the data from the KM2A half-array from the standard candle at very high energies the Crab Nebula - investigated the detector performance for gamma-rays. The measured by them statistical significance of the gamma-ray signal from the Crab Nebula is $28.0 \sigma$ at $25-100$ $\mathrm{TeV}$ and $14.7 \sigma$ at $>100 \mathrm{TeV}$ [5]. Also, the LHAASO collaboration reported about the detection of more than 530 photons at energies above $100 \mathrm{TeV}$ and up to $1.4 \mathrm{PeV}$ from 12 ultrahighenergy $\gamma$-ray sources with statistical significances greater than 7 б [6].

TAIGA (Tunka Advanced Instrument for cosmic ray physics and Gamma Astronomy) [7] includes wide-angle, Cherenkov light integrating HiSCORE detectors, IACTs and particle detectors. These three arrays are operating in coincidence. TAIGA aims to address gamma-ray astronomy at energies from a few $\mathrm{TeV}$ to several $\mathrm{PeV}$, cosmic ray physics from $100 \mathrm{TeV}$ to several $\mathrm{EeV}$, as well as to pursue search for axion-like particles, Lorentz invariance violation and other unexpected manifestations of New Physics. The novel approach of combining the IACTs of the TAIGA array with the timing wide-angle Cherenkov HiSCORE array [8] within one instrument expands the capabilities of traditional IACTs towards higher energies. Since this combined array does not require the shower detection by several IACTs, the inter-telescope distances can be essentially increased up to $600-1000 \mathrm{~m}$; please note that this is impossible for the standard stereoscopic mode of the IACT arrays. The common operation will allow us to use for the primary particle identification a combination of data processing techniques generally exploited in the analysis of both the imaging and non-imaging (timing) instruments. This costeffective experimental solution can allow one to build a large array of up to $10 \mathrm{~km}^{2}$ for studying the PeVatrons and searching for diffuse gamma rays with energy $\geq 100 \mathrm{TeV}$.

\section{The Astrophysical complex TAIGA}

At the moment, the following installations are operating as part of the complex: Tunka133, Tunka-Grande, TAIGA-HiSCORE, TAIGA-IACT, TAIGA-MUON (Fig.1). Below we briefly describe only the last four arrays from this list. All arrays are synchronized in time with each other with an accuracy of $10 \mathrm{~ns}$. The synchronization method is described in detail in [12].

\subsection{The timing wide-angle Cherenkov TAIGA-HiSCORE array}

The principle of the timing wide-angle Cherenkov TAIGA-HiSCORE array follows the idea outlined in [11]. It is similar to the one used for the Tunka-133 array [10]. Again, this method is based on the sampling of the Cherenkov light front of air showers, but a threshold of the TAIGA-HiSCORE is almost 20 times lower compared to that of the Tunka-133 array. Also, the accuracy of reconstruction of EAS parameters is much higher. The TAIGA-HiSCORE pilot 
complex includes 120 wide-angle optical stations, distributed with spacing of $106 \mathrm{~m}$ over an area of about $1 \mathrm{~km}^{2}$.

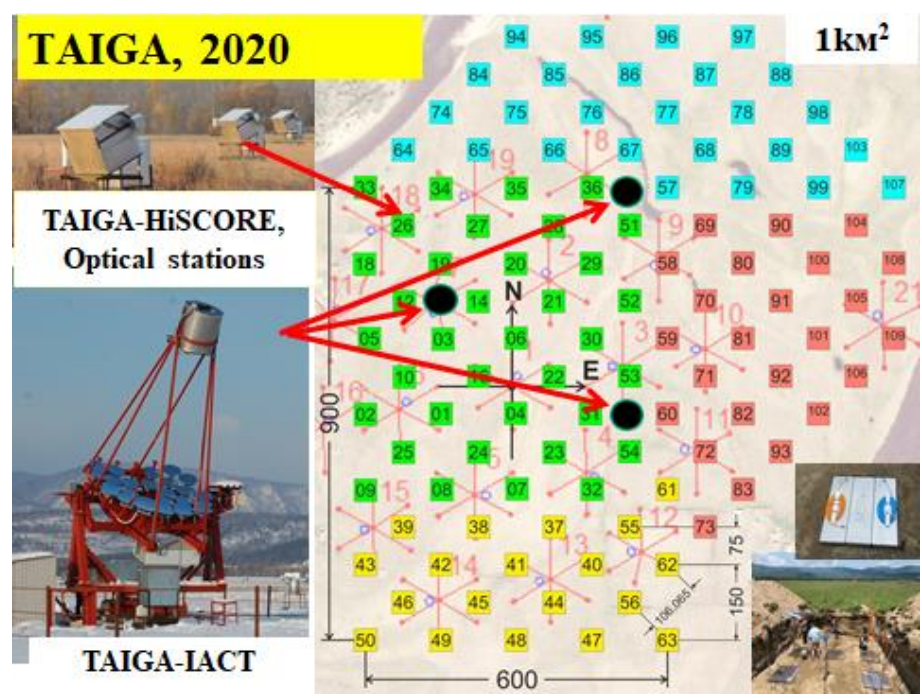

Figure 1. The topography of the TAIGA pilot complex.

The TAIGA-HiSCORE stations consist of two containers - an optical container with 4 PMTs, and a container with data acquisition electronics [12]. Hemispherical PMTs with a photocathode of 20 to $25 \mathrm{~cm}$ diameter are used. Each PMT is attached to a Winston cone with input window of $0.4 \mathrm{~m}$ diameter, which provides a field of view (FoV) of $\sim 60^{\circ}(\sim 0.6 \mathrm{sr})$. The total light collection area of one station is $0.5 \mathrm{~m}^{2}$. The PMTs, the voltage-dividers, the preamplifiers and the light concentrators are installed inside a metallic container with a protective Plexiglas cover. Underneath the latter a heating-wire prevents from humidity condensation on the window. A radio-controlled automatic sliding lid mechanism exposes the detector to light. In winter season 2020-2021 all the optical containers were tilted to the southern direction by $25^{\circ}$ to increase the observation time of the Crab Nebula. Signals from the anode and intermediate dynode are digitized with a time step of $0.5 \mathrm{~ns}$ by the 8 -channel digitizing boards based on the DRS-4 chip. Digitization of signals is carried out when the local trigger of a given station is produced. The latter is formed when the analog sum of signals from the four adjacent PMT anodes within a given HiSCORE station exceeds the preset threshold of $\sim 200$ photoelectrons (p.e.). Such threshold corresponds to the Cherenkov light flux of 0.3 photon $\mathrm{cm}^{-2}$ [13]. The optical stations of the array are grouped into 4 clusters of approximately 30 detectors each. Each optical station in a cluster is connected to the DAQ center by an optical cable for the data transmission and the synchronization. The threshold of the array for gamma-ray induced EAS is $40-50 \mathrm{TeV}$, while for cosmic rays it is $80-100 \mathrm{TeV}$ at the location height of the Tunka site.

The reconstruction of shower parameters is performed using algorithms developed for the Tunka-133 array [10,14]. The angular resolution is equal to $0.4^{\circ}-0.5^{\circ}$ for events with $4-5$ hit stations and about $0.1^{\circ}$ for events with more than 10 hit stations [9]. The primary particle energy is reconstructed using the Cherenkov light flux density at a distance of $200 \mathrm{~m}$ from the EAS axis with an accuracy of about $15 \%$. The accuracy of the reconstruction procedure was checked by using MC simulations as well as by using the experimental data.

The LIDAR instruments on the International Space Station and the Calipso satellite have been used as tools for calibrating TAIGA $[15,16]$. The measurement of the laser pulses from the mentioned satellites proved that the accuracy of absolute pointing calibration of the TAIGAHiSCORE is better than 0.1 degrees. 


\subsection{TAIGA-IACT}

Three Imaging Atmospheric Cherenkov Telescopes of the TAIGA-IACT array (Fig. 2) are installed at the vertices of a triangle with side lengths of $300 \mathrm{~m}, 400 \mathrm{~m}$ and $500 \mathrm{~m}$ approximately central within the TAIGA-HiSCORE optical stations (Fig.3). The telescopes have an alt-azimuth mount and an imaging camera in the focus of the segmented Davis-Cotton design reflector with a diameter of $4.3 \mathrm{~m}$ and a focal length of $4.75 \mathrm{~m}$.

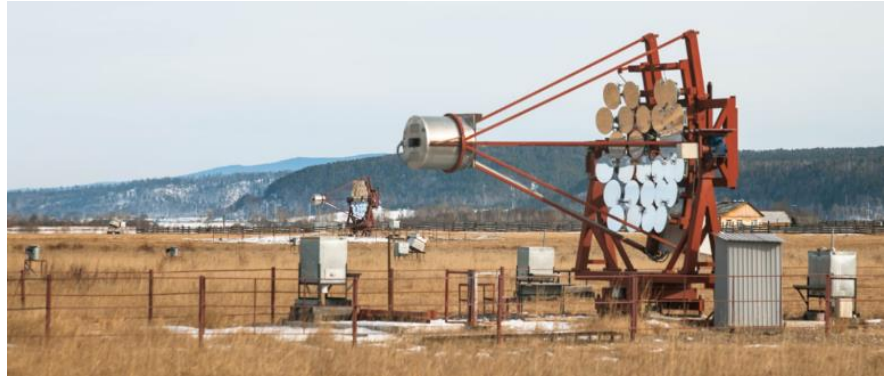

Figure 2. The two Imaging Atmospheric Cherenkov Telescopes of the TAIGA-IACT array.

The cameras of a TAIGA-IACTs [17] include a matrix of 560 PMTs for the first telescope and 595 PMTs for the second and the third telescopes. The $19 \mathrm{~mm}$ diameter PMTs of type XP1911 from Philips are used in all cameras. The FoV of the camera is $9.6^{\circ}$ (each pixel has an aperture of $0.36^{\circ}$ ), and the optical PSF of the reflector is $0.07^{\circ}$. The CCD-camera Prosilica GC1380 is installed on each of the telescopes. It is used for checking the telescope pointing direction. The imaging camera consists of identical clusters, each based on 28 PMTs. The basis of the cluster electronics is a 64-channel ASIC MAROC-3. Each channel includes a preamplifier with adjustable gain, a charge sensitive amplifier and a comparator with adjustable threshold. This chip has a multiplexed analog output signal which is proportional to the input charge. The chip is connected to a 12-bit external ADC. The signal from each PMT is split and fed into 2 MAROC-3 channels with gain differences of $x 30$. This results in a full dynamic range of 3000 p.e. The source observations were performed in the so-called "wobble mode" [18]. In this observation mode, we point the IACTs with an offset of $1.2^{\circ}$ in right ascension with respect to the source position [19].

\subsection{Tunka-Grande and TAIGA-Muon}

There are a lot of good reasons to include particle detectors in the TAIGA hybrid detector complex. In particular, the number of muons in a charged cosmic-ray induced EAS is larger than in gamma-ray events by 30 times, so measuring the muon number is a very effective way to suppress the unwanted background. The Tunka-Grande array [20] is a network of scintillation counters combined into 19 stations. Each of these can be conditionally represented as consisting 2 parts: one on the surface and another one underground. The former, consisting of 12 counters with a total area of about $8 \mathrm{~m}^{2}$, detects all charged particles of EAS at the array level. The second, consisting of 8 counters with a total area of about $5 \mathrm{~m}^{2}$, is located underground, below the soil layer of $1.5 \mathrm{~m}$ in thickness. It is designed to detect the muon component of EAS.

The total area of muon detectors in the Tunka-Grande installation is currently only $100 \mathrm{~m}^{2}$. Such an area of course is not enough for studying the mass composition in the range of $10^{16}$ $10^{17} \mathrm{eV}$, which is of the greatest interest to us. We have further increased the area of muon 
detectors with the creation of the TAIGA-Muon array [21]. New scintillation counters with an area of $1 \mathrm{~m}^{2}$ have been developed for this installation [22]. The average signal amplitude during the passage of a muon for these counters is $20-25$ p.e. In 2019 , the first three clusters of the TAIGA-Muon installation were deployed, each cluster has 8 surface and the same number of underground detectors. The counters are located in pairs, the surface ones being strictly above the underground ones. All 8 pairs are placed along the perimeter of a square with a side length of $5 \mathrm{~m}$. The distance between the adjacent pairs is $1 \mathrm{~m}$. By 2024, it is planned to increase the area of underground muon detectors in the TAIGA-Muon installation up to $400 \mathrm{~m}^{2}$.

\section{The recent results}

\subsection{The energy spectrum of cosmic rays and the mass composition}

Based on the data of TAIGA-HiSCORE and Tunka-133 the energy spectrum of cosmic rays was reconstructed in the energy range 0,3 $-1000 \mathrm{PeV}$ (Fig.3) [23,24]. There is a good agreement, both with the direct balloon [25] and the satellite [26] and the high-altitude [27] measurements at low energies, and with measurements of giant installations at extremely high energies (Pierre Auger Observatory [30], Telescope Array [31]). In all the experimental data the spectrum index decreases at the energy of $\sim(1.5-2) 10^{16} \mathrm{eV}$ by $0.2-0.3$. At present, this effect has no clear astrophysical explanation. A number of features are observed in the energy spectrum, which have not yet obtain an astrophysical interpretation. First of all, the spectrum in the region of the "first knee" at energy of 3-6 PeV cannot be interpreted as just a sharp change in the slope of the spectrum. The feature in the spectrum at about $20 \mathrm{PeV}$, which was reliably established by a number of experiments, yet could not be explained either. Finally, the energy were the "second knee" is located, is still determined with large uncertainty.

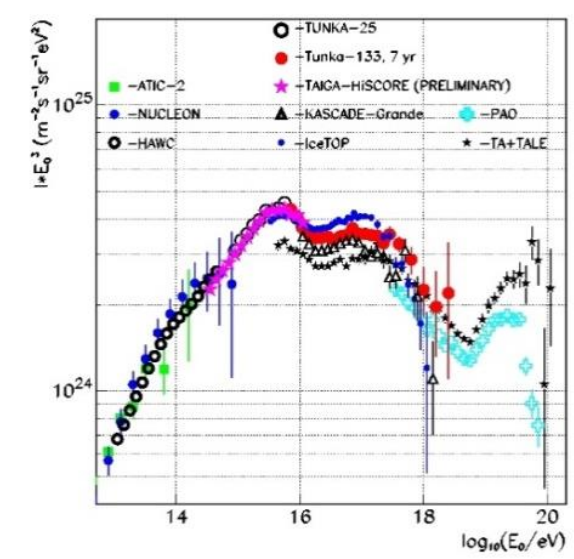

Figure 3. Comparison of the energy spectra of cosmic rays obtained in various experiments in a wide energy range: ATIC-2 [25], NUCLEON [26], HAWC [27], Tunka-25, TAIGAHiSCORE, Tunka-133, KASCADE-Grande [28], Ice-Top [29], PAO [30], TA+Tale [31]

At this conference the first preliminary results of the cosmic rays energy spectrum from the Tunka-Grande array was presented [32]. For the Tunka-Grande array, the EAS energy is determined by the particle density at a distance of $200 \mathrm{~m}$ from the EAS core position. We used 
the coincident with Tunka-133 events for the energy calibration. The presented energy spectrum is in good agreement with the energy spectrum inferred from Tunka-133.

The average depths of the EAS maxima obtained for the two installations, depending on the energy of the primary particle, are shown in Fig. 4A. The data of both installations, despite the differences in their geometry, fit well together, providing a wide energy range from 1 to 300 $\mathrm{PeV}$. Our experimental data are compared with the results of direct measurements of the maximum depth obtained by observing the fluorescent light at PAO [35].

The results of our measurements show that light nuclei - protons and helium dominate in the cosmic ray flux in the energy range of $1-100 \mathrm{PeV}$ (Fig.4, B). The dependence on the energy of mean value of LnA fits very well with the results of PAO at energy of $300 \mathrm{PeV}$ (Fig. 4 B). But still there is no satisfactory agreement on the mass composition of cosmic rays in the region of the first knee in different experiments.

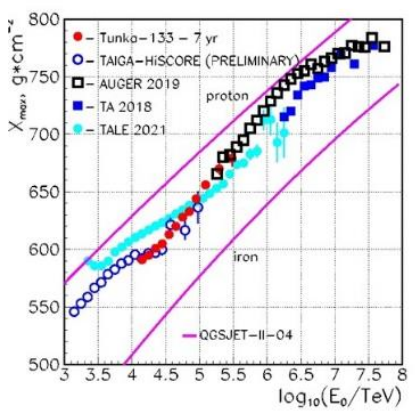

A

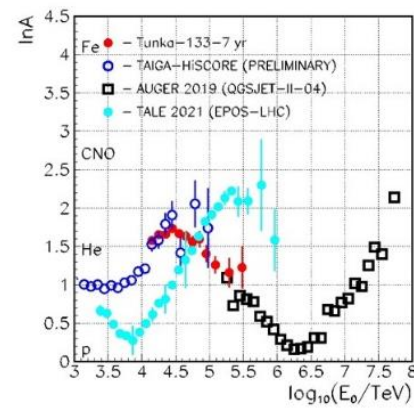

$\mathrm{B}$

Figure 4 A: Dependence of the average depth of the mean Xmax from the energy $B$ : The dependence of the average logarithm of the mass $<\ln A>$ on the energy, Tunka-133 [34] , 2 TAIGA-HiSCORE[34],3 - PAO [35], TA-TALE[36]

\subsection{Very high energy gamma-rays from the Crab Nebula}

TAIGA allows us to implement various approaches for detecting gamma-rays from local sources:

1. Stand-alone operation of a single IACTs for $\mathrm{E}<10-15 \mathrm{TeV}$

2. Stereoscopic approach for large distances between the IACTs for $\mathrm{E} \geq 10 \mathrm{TeV}$

3. Hybrid approach-1 - joint operation of HiSCORE and IACTs for $\mathrm{E} \geq 40 \mathrm{TeV}$.

4. Hybrid approach-2 - joint operation of HiSCORE and muon detectors for $\mathrm{E} \geq 500 \mathrm{TeV}$.

To date, the telescopes of the complex have passed the first exam - the signal from the Crab Nebula was measured at the significance level of 6 sigma in 40 hours of observations in the winter season 2019-20 [37]. At the TAIGA location it is possible to observe the Crab Nebula only under zenith angle $>29^{\circ}$. A zenith angle upper limit of $42^{\circ}$ was applied to the dats. The image analysis was performed using conventional technique, the so-called dynamical cuts, which means energy-dependent cuts on the image parameters introduced by M. Hillas. The set of cuts of Hillas parameters used in this analysis can be found in [37]. After cutting on the image size ( $>125$ p.e.), the energy threshold for gamma rays is $\sim 3-4 \mathrm{TeV}$. The $\alpha$ distribution of events after all cuts is presented in Fig 5. The total number of excess gamma events is 164. Monte Carlo simulations revealed good agreement with the results obtained: for the Crab Nebula, the esti- 
mated number of gamma rays after cuts is $\sim 150-200$ depending on the fit of the Crab Nebula energy spectrum.

Currently for stereoscopic analysis we can use the data of two IACT. For the two operating IACTs at the inter-telescope distance of $320 \mathrm{~m}$ the counting rate of coincident events is $\sim 1 \mathrm{~Hz}$. The data analysis is in progress. The third telescope will be put into operation in fall 2021. The 4th and the 5th IACTs will be installed in the next 2 years. The distance between the TAIGAIACTs will be at least $250 \mathrm{~m}$, the effective area for events detected by 5 telescopes simultaneously will reach $0.9 \mathrm{~km}^{2}$ at an energy of $22 \mathrm{TeV}$ [38].

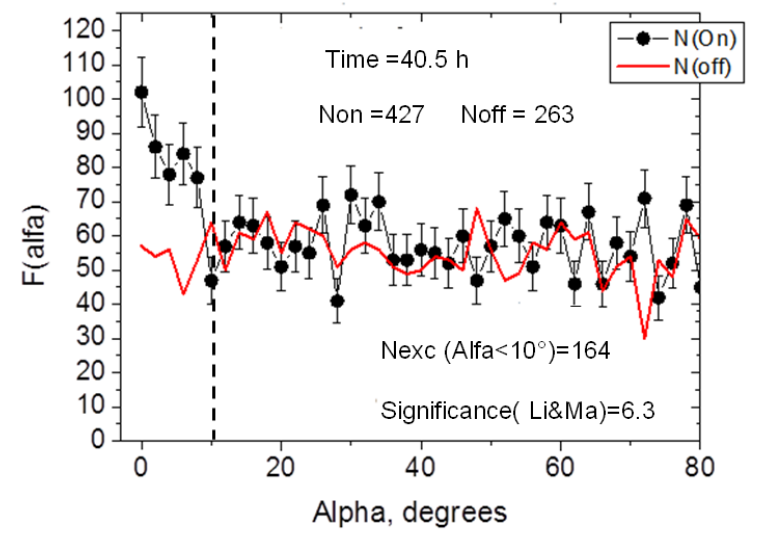

Figure 5. Distribution over the alpha parameter for observations of the Crab Nebula (On) and background (Off) events.

As part of a hybrid approach, we analyzed data from a 115-hour observation of the Crab Nebula by first IACT and two TAIGA-HiSCORE clusters (effective area $0.3 \mathrm{~km}^{2}$ ). For every event we have reconstructed: the energy, the core position, the arrival direction, the declination, the angle between shower axis and the direction to the Crab Nebula, etc. using TAIGAHiSCORE data. As the main criteria of gamma induced shower search we used the difference between the 'On' and 'Off' distributions of the Hillas parameter alpha. There were selected 4 candidates in the 'On' sample and not a single candidate in 'Off' mode. All 4 events have energy in the interval 100-170 TeV, zenith angle range 29-36 degrees, core positions inside a circle of $\mathrm{R}=320 \mathrm{~m}$ from the IACT. Detailed analysis shows that lateral distributions function for all 4 events is very flat, as it is expected for gamma induced showers.

\section{Conclusion}

In 2021 we intend to finish the deployment and the commissioning of the one square kilometer TAIGA setup (TAIGA pilot complex) in the Tunka valley, about $50 \mathrm{~km}$ from Lake Baikal in Siberia, Russia. Since 2009 the timing Tunka-133 Cherenkov EAS array has been in operation there [10]. The TAIGA pilot complex (Fig.1) will include 120 wide-angle Cherenkov detectors (optical stations) of the timing TAIGA-HiSCORE array, three IACTs of the imaging TAIGA-IACT array and 5 clusters of the scintillation counters of a TAIGA-Muon array. In the next 2 years 2 more IACT will be produced and installed. As the next step we intend to construct full-scale gamma observatory TAIGA with about 1000 timing Cherenkov detectors and 10-15 IACTs distributed on the area of $\sim 10 \mathrm{~km}^{2}$. We are looking for an observation site with suitable astronomical climate for the full-scale gamma-observatory TAIGA. 


\section{Acknowledgments}

The work was performed at the UNU "Astrophysical Complex of MSU-ISU" (agreement 13.УHУ.21.0007). The work is supported by Russian Foundation for Basic Research (grants № 19-52-44002, 19-32-60003), the Russian Science Foundation (grant 19-72-20067 (Section 3,4), the Russian Federation Ministry of Science and High Education (projects FZZE-2020-0017, FZZE-2020-0024 and FSUS-2020-0039).

\section{References}

[1] F.Aharonian (HESS Collaboration)//Astroparticle Physics 34(2011) 738-747

[2] Albert et al // Astrophys.J.639:761-765,2006 e-Print: astro-ph/0508543 MAGIC http://wwwmagic.mppmu.mpg.de/

[3] V.Acciari (VERITAS Collaboaration) Ap.J Letters 730 (2011) L20 h

[4] M. Amenomori et al. Phys. Rev. Lett. 123 (2019) arXiv:1906.05521

[5] F. Aharonian et al. Chinese Phys. C 45 (2021) 025002. arXiv:2010.06205

[6] Zhen Cao et al Nature volume 594 (2021) 33.

[7] N. Budnev et al., Nucl.Instrum.Meth. A 845 (2017) 330

[8] M Tluczykont et al., Journal of Physics: Conference Series 632 (2015) 012042

[9] L. Kuzmichev et al. (TAIGA Collaboration) EPJ Web Conf. 145 (2017) 01001

[10] Berezhnev S.F. et al.,(Tunka Collaboration) Nucl.Instrum.Meth. A 692 (2012) 98

[11] M. Tluczykont et al., Astroparticle Physics 56 (2014) 42

[12] O. Gress et al., ((TAIGA Collaboration) Nucl. Instrum. Meth. A 845 (2017) 367

[13] S. Berezhnev et al ( TAIGA Collaboration) Bull. Russ. Acad. Sci. Phys. 79, 348 (2015)

[14] V.V. Prosin et al. (Tunka Collaboration), Nucl.Instrum.Meth. A 756 (2014) 94

[15] A.Porelli et al., (TAIGA Collaboration) PoS ICRC2017, 754 (2017).

[16] A. Porelli for the TAIGA collaboration, PoS ICRC2021, 876 (2021).

[17] N. Lubsandorzhiev et al., (TAIGA Collaboration), PoS ICRC2017 757 (2017)

[18] Stepanian, A. Lamb, R., et al., Astropart. Phys. 2 (1994), no. 2, p. 137.

[19] Zhurov, D., Gress, O., Sidorov, D., et al., J. Phys.:Conf. Ser. 1181 (2019) 012045.

[20] R. D. Monkhoev et al, Bull. Russ. Acad. Sci. 81 (2017) 4, 468-470

[21] A.Ivanova et al. (TAIGA Collaboration) JINST 15 C06057 (2020)

[22] I. Astapov et al. (TAIGA Collaboration), Nucl. Instrum. Meth. A 936, 254-256 (2019)

[23] N. Budnev et al. (Tunka Collaboration) Astropart. Phys., 117, 102406, (2020)

[24] V. Prosin, et al. (TAIGA Collaboration), Bull. of the RAS: Phys., 83, 1016-1019

(2019)

[25] A. Panov, et al. Bull. of the RAS., Phys. 73, 5, 564-567 (2009)

[26 ]A. Turundaevskiy et al. Bull. of the RAS, Phys. 85, 4. 353 (2021)

[27] R. Alfaro, et al. Phys. Rev. D. 96. 122001.(2017)

[28].W.D.Apel et al. Astropart. Phys, 36, 183. (2012)

[29].M. G. Aartsent et al .Phys. Rev. D. 88. 042004. (2013)

[30]J. Abraham et al. Physics Letters B 685, 239-246 (2010)

[31].R.U. Abbasi et al. Astrophys. J. 858, 76. (2018)

[32] A.Ivanova et al (TAIGA Collaboration) PoS ICRC2021, 361 (2021).

[33] R.U. Abbasi et al. Astrophys. J. 858, 76. (2018)

[34] V. Prosin, et al., Bull. of the RAS, Phys., 85, 4, 395 - 397, (2021)

[35] . A.Yushkov et al, Proc. 36th ICRC .PoS 482, arXiv: 1909.090173 (2019)

[36] R.U. Abbasi et al. Astrophys. J. 909 2, 178 (2021)

[37] L.Sveshnikova et al.(TAIGA Collaboration) Bull. of the RAS: Phys.,85, 4, 398(2021)

[38] A.Grinyuk, E. Postnikov, P. Volchugov for the TAIGA collaboration, PoS ICRC2021, 713. 


\section{Full Authors List: TAIGA Collaboration}

I. I. Astapov ${ }^{3}$, A. K. Awad ${ }^{10}$, P. A. Bezyazeekov ${ }^{2}$, M. Blank ${ }^{10}$, E. A. Bonvech ${ }^{1}$, A. N. Borodin $^{4}$, A. V. Bulan ${ }^{1}$, M. Brückner ${ }^{5}$, N. M. Budnev' ${ }^{2}$, A. Chiavassa ${ }^{6}$, D. V. Chernov ${ }^{1}$, A. N. Dyachok $^{2}$, A. R. Gafarov ${ }^{2}$, A. Yu. Garmash ${ }^{7.8}$, V. M. Grebenyuk ${ }^{4.9}$, O. A. Gress ${ }^{2}$, E.O.

Gress $^{2}$, T. I. Gress ${ }^{2}$, O. G. Grishin ${ }^{2}$, A. A. Grinyuk ${ }^{4}$, D. Horns ${ }^{10}$, A. Igoshin ${ }^{1}$, A. D. Ivanova ${ }^{2}$, A. L. Ivanova, ${ }^{7,2}$, N. N. Kalmykov ${ }^{1}$, V. V. Kindin ${ }^{3}$, S. N. Kiryuhin ${ }^{2}$, R. P. Kokoulin ${ }^{3}$, K. G. Kompaniets $^{3}$, E. E. Korosteleva ${ }^{1}$, V. A. Kozhin ${ }^{1}$, E. A. Kravchenko ${ }^{7.8}$, A. P. Kryukov ${ }^{1}$, L. A. Kuzmichev $^{1}$, A. A. Lagutin ${ }^{11}$, M. Lavrova ${ }^{4}$, Yu.E. Lemeshev ${ }^{2}$, B. K. Lubsandorzhiev ${ }^{12}$, N. B. Lubsandorzhiev $^{1}$, A. D. Lukanov ${ }^{12}$, D. S. Lukyantsev' ${ }^{2}$, S.D. Malakhov ${ }^{2}$, R. R. Mirgazov ${ }^{2}$, R. Mirzoyan $^{13,1}$, R. D. Monkhoev ${ }^{2}$, E. A. Osipova ${ }^{1}$, A. L. Pakhorukov ${ }^{2}$, A. Pan ${ }^{4}$, L. Panasenko ${ }^{7}$, L. V. Pankov ${ }^{2}$, A. D. Panov ${ }^{1}$, A. A. Petrukhin ${ }^{3}$, I.A. Poddubny ${ }^{2}$, D. A. Podgrudkov', V. A. Poleschuk $^{2}$, V. Ponomareva ${ }^{2}$, M. Popesku ${ }^{14}$, E. G. Popova ${ }^{1}$, A. Porelli ${ }^{5}$, E. B. Postnikov ${ }^{1}$, V.V. Prosin ${ }^{1}$, V. S. Ptuskin ${ }^{15}$, A. A. Pushnin ${ }^{2}$, R. I. Raikin ${ }^{11}$, A. Y. Razumov ${ }^{1}$, G. I. Rubtsov ${ }^{12}$, E. V. Ryabov ${ }^{2}$, Y. I. Sagan ${ }^{4.9}$, V. S. Samoliga ${ }^{2}$, A. A. Silaev ${ }^{1}$, A. A. Silaev(junior) ${ }^{1}$, A. Yu. Sidorenkov $^{12}$, A. V. Skurikhin ${ }^{1}$, M. Slunecka ${ }^{4}$, A. V. Sokolov ${ }^{7.8}$, Y.V. Suvorkin ${ }^{2}$, L. G. Sveshnikova $^{1}$, V. A. Tabolenko ${ }^{2}$, A.B. Tanaev ${ }^{2}$, B. A. Tarashansky ${ }^{2}$, M.Yu. Ternovoy ${ }^{2}$, L. G. Tkachev $^{4.9}$, R. Togoo ${ }^{16}$, M.Tluczykont ${ }^{10}$, N. Ushakov ${ }^{12}$, A. Vaidyanathan ${ }^{7}$, P. A. Volchugov ${ }^{1}$, N. V. Volkov ${ }^{11}$, D. Voronin ${ }^{12}$, R. Wischnewski ${ }^{5}$, A. V. Zagorodnikov ${ }^{2}$, A.D. Zhaglova ${ }^{2}$, D. P. Zhurov $^{2.17}$, I. I. Yashin ${ }^{3}$

1 Skobeltsyn Institute ofNuclear Physics, MoscowStateUniversity, Moscow, 119991 Russia. 2 Research Institute of Applied Physics, Irkutsk State University, Irkutsk, 664003 Russia.

3 National ResearchNuclearUniversity MEPhI, Moscow, 115409 Russia.

4 Joint Institute for Nuclear Research, Dubna, Moscow oblast, 141980 Russia.

5 Deutsches Elektronen-Synchrotron DESY, Zeuthen, 15738 Germany.

6 Physics Department of the University of Torino and Istituto Nazionale di Fisica Nucleare, Torino, 10125 Italy.

7 Novosibirsk State University, Novosibirsk, 630090 Russia.

8 Budker Institute of Nuclear Physics, Siberian Branch, Russian Academy of Sciences, Novosibirsk, 630090 Russia.

9 Dubna University, Dubna, Moscow oblast, 141980 Russia.

10 Institut für Experimentalphysik, Universität Hamburg, Hamburg, D-22 761 Germany.

11 Altai State University, Barnaul, Altai krai, 656049 Russia.

12 Institute for Nuclear Research, Russian Academy of Sciences, Moscow, 117312 Russia.

13 Max Planck Institute for Physics, Munich, 80805 Germany.

14 Space Science Institute, Magurele, 077125 Romania.

15 Pushkov Institute of Terrestrial Magnetism, Ionosphere and Radio Wave Propagation, Russian Academy of Sciences, Troitsk, Moscow, 142190 Russia.

16 Institute of Physics and Technology Mongolian Academy of Sciences, Ulaanbaatar, Mongolia.

17 Irkutsk National Research Technical University, Irkutsk, Russia. 